\title{
Critical damping in a kinetic interaction network
}

\author{
Derek A. Paley and Ajay K. Baharani
}

\begin{abstract}
In a kinetic interaction network, signals are emitted through motion. Natural examples include bird flocks, fish schools, and robot teams. A kinetic interaction network transmits information about external cues quickly and accurately. Analysis of a one-dimensional interaction network reveals a bound on the algebraic connectivity above which the transient response is overdamped. A critically damped response, the fastest and most accurate, is achieved by maximizing the algebraic connectivity subject to this bound. For example, in an $n$-neighbor interaction network, output rise time is minimized for intermediate values of $n$. This analytical result yields insight into natural networks and a design method for synthetic networks. We apply this result to automobile congestion by extending the Intelligent Driver Model to include interactions with multiple vehicles. Simulations indicate that, in certain portions of parameter space, traffic flow is improved by including directed interactions with an intermediate number of vehicles.
\end{abstract}

\section{INTRODUCTION}

A distributed network of unmanned vehicles that interact through relative motion is capable of coordinated behavior, even without directed communication. We apply linear systems theory and algebraic graph theory to model the transmission of information in a communication network in which signals are emitted through motion. We use this model to identify the topological configurations that achieve a critically damped response to an internal or external cue. The primary goal is to provide a theoretical foundation for optimizing a motion-based interaction network for speed and accuracy of information transmission. Progress towards this goal is highlighted below in an application of the main result to automobile congestion. A secondary goal is to gain insight into naturally occurring interaction networks. Progressing towards the second goal is ongoing and outside the scope of the present paper.

A natural kinetic interaction network - such as a bird flock, fish school, or human crowd-reacts almost simultaneously to an exogenous threat or endogenous event. For example, a flock of birds will turn in unison to avoid a predator or roost in a tree. The collective response of a bird flock or a fish school is the product of a fast-moving information wave that propagates when neighboring individuals react to changes in relative position and velocity [1].

A remarkable property of a natural interaction network is the inherent capability to accurately and rapidly transmit

D. Paley is an assistant professor in the Department of Aerospace Engineering, University of Maryland, College Park, MD USA dpaley@umd. edu

A. Baharani is a graduate student in the Department of Aerospace Engineering, University of Maryland, College Park, MD USA ajay.baharani@gmail.com information about internal or external cues that are sensed by only a few individuals. In a fish school, this phenomenon, known as a "wave of agitation", propagates faster than the speed of an individual fish [1] and enables the school to respond as one to an external threat. Since fish do not communicate verbally, the signaling is accomplished primarily by movement (kinetics). Just like a driver in automobile traffic, each individual regulates the range and range rate to neighboring individuals that encroach or move away.

The dynamics of interaction networks have been primarily described by empirical studies in biology and theoretical studies in physics and engineering. Empirical studies show that schooling fish exhibit more accurate responses to external threats than individual fish [2]. Empirical studies of starlings suggest that topological distance, as opposed to metric distance, may determine the interaction network in bird flocks [3]. In topological-distance coupling, the strength of interaction between two individuals is determined not by their physical separation but by the number of other individuals between them. Starling-flock observations show that anisotropy of neighbor positions disappears above an average node degree of 6.5 independent of density [3]. These observations motivate our investigation of $n$-neighbor networks-in which each node interacts with its $n$ closest neighbors-rather than proximity graphs-in which interactions occur over a finite range.

Theoretical analyses of network dynamics have primarily emphasized steady-state responses such as consensus and collective motion. Under very mild assumptions on the interaction topology, consensus is achieved by simple, decentralized algorithms [4], [5]. The rate of convergence to consensus can be increased by maximizing the algebraic connectivity of the network, which is the smallest nonzero eigenvalue of the Laplacian matrix [6]. Fast consensus can be achieved in small-world networks [7] or by optimization of the edge weights [8], [9]. Activity consensus such as moving in the same direction or orbiting a common point can be achieved by decentralized algorithms in synthetic networks with time-varying or time-invariant and directed or undirected communication topologies [10], [11], [12], [13].

This paper considers the transient response of an interaction network by applying systems and graph theory to analyze an idealized model of an interaction network. We use elementary physical components-point masses, linear springs, and linear dampers - to construct a one-dimensional network of harmonic oscillators. (A spring is used to model an attractive/repulsive spacing force and a damper is an idealized representation of the effect of velocity matching.) The oscillator network can be viewed as an single-input, single- 
output system, where the input is a force on one mass and the output is the position of another. Spectral analysis relates the transient response of the network to the topology of the interaction graph. Despite its simplifying assumptions, this framework provides a methodology for optimizing synthetic networks for fast and accurate information transmission.

Analysis of the one-dimensional model characterizes the network's transient response in terms of the algebraic connectivity of the interaction graph and damping ratio of the oscillator interactions. Specifically, we observe that a critically damped network response occurs when the algebraic connectivity is maximized subject to a constraint inversely proportional to the squared damping ratio. This result implies that the fastest and most accurate response of a nearestneighbor network will occur when the neighbor interactions are limited to intermediate topological distances-which is consistent with quantitative analysis of starling flocks [3]. (The topological distance between agent $k$ and its $n$th closest neighbor is $n$.) For the specific case of a nearest-neighbor ring, we identify the number of neighbors that achieves a critically damped response as a function of the damping ratio and network size.

We highlight the broad applicability of the main result by studying a nonlinear model of automobile-driver interactions, specifically the Intelligent Driver Model (IDM) [14]. IDM models acceleration and braking behavior of an individual driver in response to a single vehicle directly in front of it. We extend the model to include interactions with multiple vehicles in front of the driver. We then conduct a parametric study of the extended IDM that characterizes the response of individual vehicles in a line of traffic, modeled as a directed $n$-neighbor network. Simulations of the extended IDM indicate that, indeed, there exists an intermediate number of neighbors $(n>1)$ that minimizes latency in traffic flow.

The paper is organized as follows. Section II describes the mathematical model of a one-dimensional kinetic interaction network. Section III presents theoretical analysis of the model and numerical simulations that validate and extend the analysis. Section IV highlights the application of the main result to a nearest-neighbor network. Section V describes the results of numerical simulations of the extended IDM. Section VI summarizes the contributions of the paper and indicates topics of ongoing research.

\section{DYNAMIC MODEL OF INTERACTION NETWORK}

We study a one-dimensional network of $N$ unit masses in which an arbitrary number of masses are coupled pairwise by identical damped springs (see Figure 1). We assume that the interaction graph is connected and undirected. In coordinates that represent the deviation of the mass positions from equilibrium, the equations of motion can be expressed in linear state-space form. We use this model to characterize the transient response of the network using the damping ratio of the node-level interactions.

The position of mass $k \in\{1, \ldots, N\}$ is denoted by $x_{k} \in$ $\mathbb{R}$ and its velocity by $\dot{x}_{k} \in \mathbb{R}$. If mass $j$ and $k$ are connected, then there exists between them a linear spring with spring

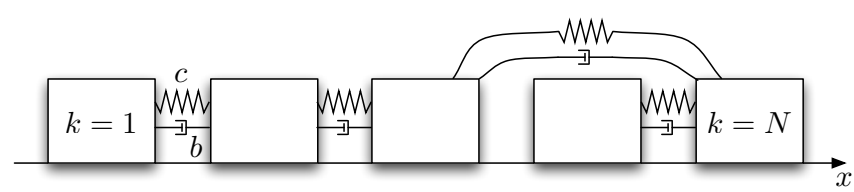

Fig. 1. One-dimensional model of an arbitrary, connected network of unit masses coupled by identical damped springs with spring constant $c$ and damping coefficient $b$.

constant $c>0$ and rest length $|k-j| x_{0}$ and a linear damper with damping coefficient $b=2 \xi \sqrt{c}$, where $\xi>0$ is the damping ratio. (The damping ratio determines the impulse response of an isolated pair of connected masses: $\xi<1$ is underdamped, $\xi>1$ is overdamped, and $\xi=1$ is critically damped.) We assume that $x_{1}(0)<x_{2}(0)<\ldots<x_{N}(0)$ and the masses do not collide.

In the case that mass $k$ is connected to only one or two other masses that are immediately adjacent to it at $t=0$, the equations of motion are

$$
\begin{aligned}
\ddot{x}_{1}= & -c\left(x_{1}-x_{2}-x_{0}\right)-2 \xi \sqrt{c}\left(\dot{x}_{1}-\dot{x}_{2}\right) \\
\ddot{x}_{k}= & -c\left(x_{k}-x_{k-1}-x_{0}\right)-2 \xi \sqrt{c}\left(\dot{x}_{k}-\dot{x}_{k-1}\right) \\
& -c\left(x_{k}-x_{k+1}+x_{0}\right)-2 \xi \sqrt{c}\left(\dot{x}_{k}-\dot{x}_{k+1}\right), \\
& k=2, \ldots, N-1 \\
\ddot{x}_{N}= & -c\left(x_{N}-x_{N-1}-x_{0}\right)-2 \xi \sqrt{c}\left(\dot{x}_{N}-\dot{x}_{N-1}\right) .
\end{aligned}
$$

Next we consider a more general coupling topology. Let $\mathcal{N}_{k}$ denote the set of masses connected to mass $k$. (The cardinality of $\mathcal{N}_{k}$ is denoted $\# \mathcal{N}_{k}$.) In the case that each mass is connected to an arbitrary set of neighbors, the equations of motion are

$$
\begin{gathered}
\ddot{x}_{k}=\sum_{j \in \mathcal{N}_{k}}-c\left(x_{k}-x_{j}-(k-j) x_{0}\right)-2 \xi \sqrt{c}\left(\dot{x}_{k}-\dot{x}_{j}\right),(1) \\
k=1, \ldots, N .
\end{gathered}
$$

Proposition 1: An equilibrium solution of (1) is $\dot{x}_{k}=$ $\dot{x}_{j}=0$ and $x_{k}=x_{j}+(k-j) x_{0}$ for all pairs of connected masses $j$ and $k$.

We write (1) in matrix notation using the $N \times N$ Laplacian matrix, $L$, of the interaction graph. Let $L_{k k}=\# \mathcal{N}_{k}$ and, for $j \neq k, L_{k j}=-1$ if $j \in \mathcal{N}_{k}$ or else $L_{k j}=0$. Note $L$ is undirected and, by assumption, connected. We have ${ }^{1}$

$$
\frac{d}{d t}\left[\begin{array}{c}
\mathbf{x} \\
\dot{\mathbf{x}}
\end{array}\right]=\left[\begin{array}{cc}
\mathbf{0 0}^{T} & I \\
-c L & -2 \xi \sqrt{c} L
\end{array}\right]\left[\begin{array}{c}
\mathbf{x} \\
\dot{\mathbf{x}}
\end{array}\right]+\left[\begin{array}{c}
\mathbf{0} \\
L \mathbf{i}
\end{array}\right] c x_{0},
$$

where $\mathbf{0}=[0, \ldots, 0]^{T} \in \mathbb{R}^{N}, I$ is the $N \times N$ identity matrix and $\mathbf{i}=[1,2, \ldots, N-1, N]^{T}$.

In order to analyze the behavior of solutions to (2), we view the mass network as a single-input, single-output system, where the input is a force on mass $k=1$ and the output is the position of mass $k=N$. In state-space form with state vector $\mathbf{z}=\left[\mathbf{x}^{T}, \dot{\mathbf{x}}^{T}\right]^{T}$, input $u$, and output $y=x_{N}$, we have the affine system

$$
\begin{aligned}
\dot{\mathbf{z}} & =A \mathbf{z}+B u+\mathbf{f} \\
y & =C^{T} \mathbf{z},
\end{aligned}
$$

\footnotetext{
${ }^{1}$ We use bold to denote an $N \times 1$ matrix, e.g., $\mathbf{x}=\left[x_{1}, \ldots, x_{N}\right]^{T}$.
} 
where

$$
A=\left[\begin{array}{cc}
\mathbf{0 0}^{T} & I \\
-c L & -2 \xi \sqrt{c} L
\end{array}\right],
$$

$\mathbf{f}=\left[\mathbf{0}^{T},(L \mathbf{i})^{T}\right]^{T} c x_{0}$ and $B$ (resp. $C$ ) is an $2 N \times 1$ matrix of all zeros except for the $(N+1)$ th (resp. $N$ th) entry, which is one.

Since there exists an equilibrium solution of (3) with $u=$ 0 , we can perform a change of coordinates to eliminate the affine term. Let $\overline{\mathbf{z}}$ denote the equilibrium solution, which implies

$$
\dot{\overline{\mathbf{z}}}=A \overline{\mathbf{z}}+\mathbf{f}=\mathbf{0} .
$$

Introducing the coordinates $\zeta=\mathbf{z}-\overline{\mathbf{z}}$ to represent the deviation of the state from the equilibrium yields

$$
\dot{\mathbf{z}}=A \mathbf{z}+B u+\mathbf{f}=\dot{\boldsymbol{\zeta}}+\dot{\overline{\mathbf{z}}}=\dot{\boldsymbol{\zeta}}
$$

and

$\dot{\boldsymbol{\zeta}}=A(\boldsymbol{\zeta}+\overline{\mathbf{z}})+B u+\mathbf{f}=A \boldsymbol{\zeta}+B u+A \overline{\mathbf{z}}+\mathbf{f}=A \boldsymbol{\zeta}+B u$.

In the new coordinates, the output is

$$
y=C^{T}(\boldsymbol{\zeta}+\overline{\mathbf{z}})=C^{T} \boldsymbol{\zeta}+\bar{x}_{N},
$$

which implies

$$
C^{T} \boldsymbol{\zeta}=x_{N}-\bar{x}_{N}
$$

In linear state-space form with state vector $\zeta$, input $u$, and output $\gamma=x_{N}-\bar{x}_{N}$, we have

$$
\begin{aligned}
\dot{\boldsymbol{\zeta}} & =A \boldsymbol{\zeta}+B u \\
\gamma & =C^{T} \boldsymbol{\zeta}
\end{aligned}
$$

where $A, B$, and $C$ are defined as before. The state variable $\zeta$ represents an $N \times 1$ matrix of the perturbations of each mass from equilibrium. We study the behavior of solutions to (5) next.

\section{SPECTRAL ANALYSIS OF NETWORK RESPONSE}

\section{A. Theoretical results for a general network}

In this section we show that the critically damped response of the model (5) to an initial displacement of mass $k=1$ minimizes the rise time without overshoot of the displacement of mass $k=N$. Solutions to the network model oscillate when the matrix $A$ defined in (4) has eigenvalues with nonzero imaginary parts. (We associate oscillations with inaccuracy in the transient response.) By solving for the eigenvalues of $A$ as a function of the eigenvalues of the Laplacian matrix $L$ and damping ratio $\xi$, we find that a critically damped response will occur when the algebraic connectivity of $L$ is maximized subject to an upper bound. Increasing the algebraic connectivity above this bound reduces the speed of the transient response due to overdamping at the network level. For the specific case of an nearestneighbor chain, we validate the analytical prediction with a numerical simulation.

Solutions to (5) will overshoot the steady-state response if there are eigenvalues of the system matrix $A$ with nonzero imaginary part. We identify the set of critically damped solutions by determining the parameter configurations in which the eigenvalues of $A$ have zero imaginary part. Suppose $\mathbf{v}=\left[\mathbf{v}_{1}^{T}, \mathbf{v}_{2}^{T}\right]^{T}$ is an eigenvector of $A$ associated with eigenvalue $\lambda$ [15]. Then

$$
\left[\begin{array}{cc}
\mathbf{0 0} & I \\
-c L & -2 \xi \sqrt{c} L
\end{array}\right]\left[\begin{array}{l}
\mathbf{v}_{1} \\
\mathbf{v}_{2}
\end{array}\right]=\lambda\left[\begin{array}{l}
\mathbf{v}_{1} \\
\mathbf{v}_{2}
\end{array}\right]
$$

which implies

$$
\begin{aligned}
\mathbf{v}_{2} & =\lambda \mathbf{v}_{1} \\
-c L \mathbf{v}_{1}-2 \xi \sqrt{c} L \mathbf{v}_{2} & =\lambda \mathbf{v}_{2} .
\end{aligned}
$$

Substituting (6) into (7) and rearranging yields

$$
L \mathbf{v}_{1}=\frac{-\lambda^{2}}{c+2 \xi \sqrt{c} \lambda} \mathbf{v}_{1} .
$$

We observe that $\mathbf{v}_{1}$ is an eigenvector of $L$ with eigenvalue

$$
\mu=\frac{-\lambda^{2}}{c+2 \xi \sqrt{c} \lambda} .
$$

Note, the eigenvalues of $L$ are real and satisfy $\mu_{1}=0<$ $\mu_{2} \leq \mu_{3} \leq \ldots \leq \mu_{N}$ [16]. Equation (8) is a quadratic formula in $\lambda$,

$$
\lambda^{2}+2 \xi \mu \sqrt{c} \lambda+c \mu=0,
$$

which has solutions

$$
\lambda=-\xi \mu \sqrt{c} \pm \sqrt{\mu c\left(\xi^{2} \mu-1\right)} .
$$

Inspection of the discriminant of (9) yields the following result.

Theorem 1: Consider the matrix $A$ is defined in (4), where $L$ is the Laplacian matrix of an undirected and connected graph with algebraic connectivity $\mu_{2}$. The eigenvalues of (4) are complex if and only if $\mu_{2}<1 / \xi^{2}$.

Proof: Using (10), an eigenvalue $\lambda$ of $A$ is complex whenever $\mu c\left(\xi^{2} \mu-1\right)<0$. Since $\mu c \geq 0$, then $\lambda$ is complex if at least one eigenvalue of the Laplacian matrix satisfies $\mu<1 / \xi^{2}$. Since $\mu_{2}$ is the smallest nonzero eigenvalue of $L$, then $\mu_{j}<1 / \xi^{2}, 3 \leq j \leq N$, implies $\mu_{2}<1 / \xi^{2}$.

If at least one eigenvalue of $A$ is complex, then the zeroinput solutions to (5) exhibit oscillations. Theorem 1 implies that there exists a bifurcation point at $1 / \xi^{2}$ that separates the oscillating (underdamped) solutions to (5) from the nonoscillating (overdamped) solutions. The bifurcation parameter is the algebraic connectivity of $L, \mu_{2}$. Although increasing the algebraic connectivity increases the rate of convergence to consensus [6], a critically damped transient response, which is the fastest and most accurate transient response, occurs when the algebraic connectivity is maximized subject to the constraint $1 / \xi^{2}$.

\section{B. Numerical validation for a n-neighbor network}

In this section we validate the theoretical predictions of the previous section using numerical simulations of an $n$-neighbor network. The simulation results illustrated in Figure 2 reveal that the output rise time is minimized by the $n$-neighbor chain with the largest algebraic connectivity that satisfies $\mu_{2}<1 / \xi^{2}$. In an $n$-neighbor chain, $n$ is an even integer and node $k$ is connected to node $j \neq k$ if 


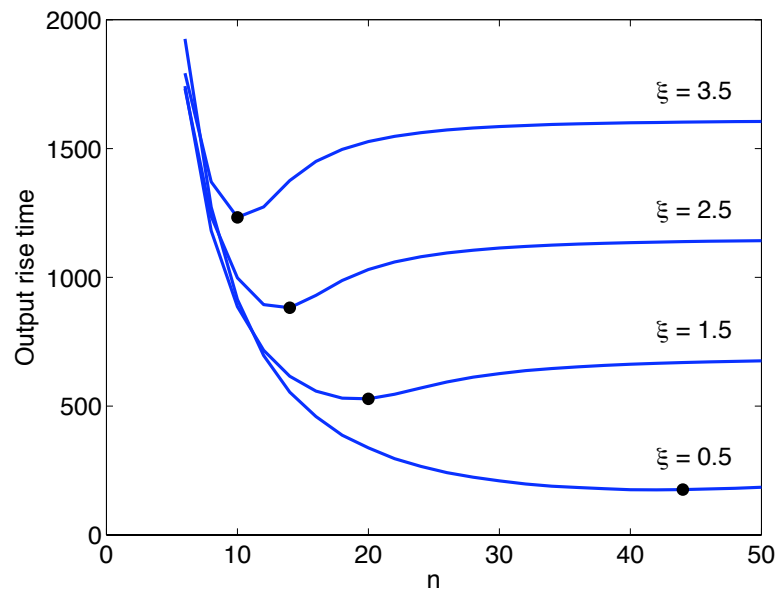

Fig. 2. Critical damping minimizes output rise time in an $n$-neighbor chain. (Results are plotted for $N=100$.) The black dots indicate the value $n=n(\xi, N)$ that maximizes the algebraic connectivity subject to constraint $\mu_{2}<1 / \xi^{2}$.

$j \in\{k-n / 2, k-n / 2+1, \ldots, k+n / 2-1, k+n / 2\}$. We integrate (5) to evaluate the transient response of $N$ masses connected in an $n$-neighbor chain. Masses $k=2, \ldots, N$ are initially in equilibrium and mass $k=1$ is initially displaced by $\delta$. The output rise time is the time elapsed from $t=0$ until the displacement of mass $k=N$ reaches $90 \%$ of its steady-state value $\delta / N$. (The steady-state value can be found by conservation of linear momentum.) Figure 2 shows that, for each damping ratio $\xi$ and network size $N$, there exists a value of $n=n(\xi, N)$ that minimizes the output rise time. In addition, the same value of $n$ generates the largest algebraic connectivity satisfying the constraint $\mu_{2}<1 / \xi^{2}$. This result suggests a network-design procedure for maximizing speed and accuracy by critically damping the transient response.

\section{NETWORK DESIGN FOR CRITICAL DAMPING}

Recall that the only assumptions on the network topology invoked in Theorem 1 are that the interaction graph is undirected and connected. As such, the result can be applied to a broad family of networks. Here, we apply the result of Theorem 1 to the design of a critically damped $n$-neighbor ring, which has a topology described by a circulant graph of degree $n$. (A circulant graph has a circulant Laplacian matrix, which implies that analytical expressions exist for the eigenstructure [17].) The goal is to find an analytical expression for the value $n=n(\xi, N)$ that maximizes $\mu_{2}$ subject to the constraint $\mu_{2}<1 / \xi^{2}$. In an $n$-neighbor network $\mu_{2}$ increases with increasing $n$. The existence of an upper bound on $\mu_{2}$ implies that, in a critically damped $n$-neighbor network, $n<N$.

The algebraic connectivity of an $n$-neighbor ring with $N$ nodes is ${ }^{2}[18]$

$$
\mu_{2}=n+1-\frac{\sin ((n+1) \pi / N)}{\sin (\pi / N)} .
$$

\footnotetext{
${ }^{2}$ Also known as an $(N, n)$ regular lattice
}

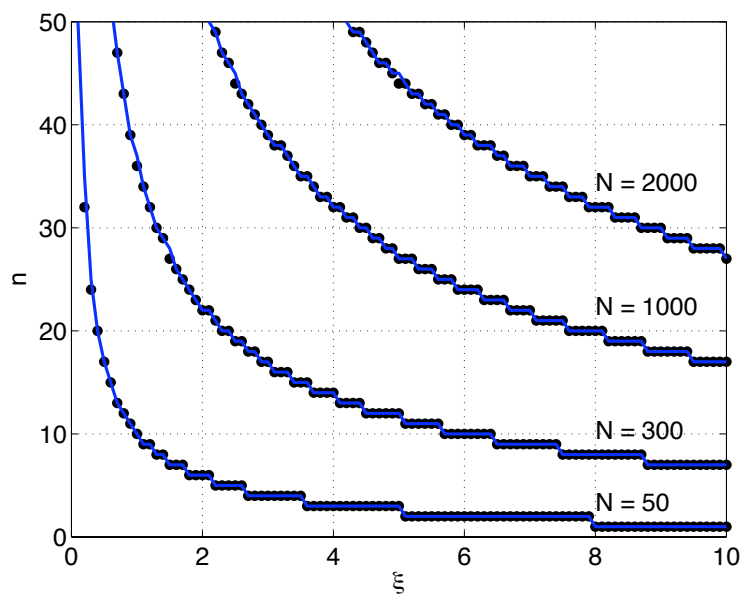

Fig. 3. The value of $n=n(\xi, N)$ that yields critical damping in an $n$-neighbor ring. Plot shows agreement between approximate analytical solution (dots) and direct numerical solutions (lines).

We seek to find the largest $n=n(\xi, N)$ satisfying the transcendental inequality

$$
n+1-\frac{\sin ((n+1) \pi / N)}{\sin (\pi / N)}<\frac{1}{\xi^{2}} .
$$

Assuming that $(n+1) \pi / N<1$, we obtain a third-order polynomial approximation of (11) by truncating the Taylor series of $\sin ((n+1) \pi / N)$ and $\sin (\pi / N)$. The third-order approximation of (11) with the inequality replaced by equality is

$$
y^{3}-y+c=0
$$

where

$$
c=-\frac{6 \sin (\pi / N)}{\xi^{2}(\pi / N)^{3}} .
$$

If (12) has a single real root, $y$, then an approximate solution to (11) is one fewer than the largest integer less than or equal to the root, i.e. $n=\lfloor y-1\rfloor$. We have the following result.

Lemma 1: The solution to the cubic formula (12) is

$$
y=\frac{1}{3 u}+u, u=\sqrt[3]{-\frac{c}{2} \pm \sqrt{\frac{c^{2}}{4}-\frac{1}{27}}}
$$

If $N^{3}|\sin (\pi / N)| / \xi^{2}>\pi^{3} /(9 \sqrt{3})$, then (12) has one real root.

Proof: The solutions to (12) can be found by Cardano's method [19]. Equation (12) has one real root if the discriminant is negative and the discriminant is $4-27 c^{2}$. Substituting $c$ defined in (12) yields the stated condition on $N$ and $\xi$.

Lemma 1 provides an approximate expression for the number of neighbors $n=n(\xi, N)$ that achieve a critically damped transient response in an $n$-neighbor ring. Figure 3 shows agreement between this approximation and a direct numerical solution to (11). The optimal number of neighbors is indeed significantly less than the total number of nodes, provided that the total number of nodes is sufficiently large and the individual interactions are not underdamped. This example highlights the use of Theorem 1 in the context 
of designing an interaction network for optimal speed and accuracy of the transient response. It also appears consistent with quantitative analysis of interaction networks in animal groups [3].

\section{Application to the Intelligent Driver Model}

A roadway traffic system inherently behaves as a motionbased interaction network. In this section we use numerical simulation of the nonlinear model to investigate the applicability of the theory to an automobile traffic system. Information flows through the network by means of visual cues that are taken in by each driver. This information is used by the driver to make a decision about his position and velocity relative to the drivers surrounding him. Inefficient transmission of this information may lead to traffic jams and backups. Traffic jams occur frequently in society and usually take some time to clear up until traffic is flowing smoothly again. If the same result seen in the simple massspring-damper simulation is observed in a traffic simulation, it implies an optimum traffic network architecture in which information is transmitted the fastest and most accurately. The results may provide more insight on how to allow traffic to flow more efficiently.

The following equations identify a traffic model known as the Intelligent Driver Model (IDM), developed and described in [14]:

$$
\begin{array}{r}
\frac{d v}{d t}=a\left[1-\left(\frac{v}{v_{0}}\right)^{\delta}-\left(\frac{s^{*}}{s}\right)^{2}\right], \\
s^{*}=s_{0}+v T+\frac{v \Delta v}{2 \sqrt{a b}},
\end{array}
$$

$a$ : maximum acceleration

$v$ : current velocity

$v_{0}$ : desired velocity

$b$ : deceleration constant

$s^{*}$ : desired gap between vehicles

$s$ : actual gap between vehicles

$s_{0}$ : minimum gap between vehicles (i.e. when $\mathrm{v}=0$ )

$T$ : desired time headway between vehicles

$\Delta v$ : velocity difference (positive when approaching)

$\delta$ : acceleration exponent

Note, equation (13) only models the longitudinal dynamics (i.e., the acceleration and braking actions) of the drivers in a one-dimensional model. This implies that there are no lane changes. The IDM assumes that velocity and position differences, $\Delta v$ and $s$ respectively, are taken with respect to the vehicle directly in front, creating a chain of drivers. Taking the leading vehicle as driver $k=1$, each of the $k$ th drivers actions solely depend on the driver immediately in front, $k-1$ for $k=2, \ldots, N$ where $N$ is the total number of drivers. The acceleration exponent $\delta$ specifies how the acceleration decreases when approaching the desired velocity [14]: the limiting case $\delta \rightarrow \infty$ corresponds to approaching $v_{0}$ with a constant acceleration $a$, whereas $\delta=1$ corresponds to an exponential relaxation to $v_{0}$.
Here we extend the IDM to account for the interactions in a directed $n$-neighbor network where each vehicle bases its actions on $n$ vehicles in front of it. The extended model (in notation consistent with the previous sections) is

$$
\begin{array}{r}
\ddot{x}_{k}=a\left[1-\left(\frac{\dot{x}_{k}}{v_{0}}\right)^{\delta}-\sum_{j \in \mathcal{N}(k)}\left(1-\left(\frac{x_{k, j}^{*}}{x_{j}-x_{k}}\right)^{2}\right)\right], \\
x_{k, j}^{*}=|k-j|\left(x_{0}+\dot{x}_{k} T\right)+\frac{\dot{x}_{k}\left(\dot{x}_{k}-\dot{x}_{j}\right)}{2 \sqrt{a b}},
\end{array}
$$

$x_{k}$ : position of the $k$ th driver

$x_{k, j}^{*}$ : desired distance between $k$ th and $j$ th drivers

$x_{0}$ : minimum gap between drivers

$\mathcal{N}(k)$ : the neighbor set of vehicle $k$

By inspecting equation (14) it can be seen that the accelerations are now the result of the sum of the interactions of the $k$ th driver with several other drivers that are contained in its neighbor set $\mathcal{N}(k)$. All vehicles (excluding the leader) have interactions with other vehicles. The leading vehicle is a unique case in this example because it has zero neighbors. Therefore its dynamics are different and can be simplified to

$$
\ddot{x}_{1}=a\left[1-\left(\frac{\dot{x}_{1}}{v_{0}}\right)^{\delta}\right] .
$$

We numerically integrated the extended IDM to see if there exists an optimum number of neighbors that minimizes the output rise time as was observed earlier in the massspring-damper model. The output used here is the actual gap between the last vehicle in the chain $(k=N)$ and the leading vehicle $(k=1)$. It is expected that the gap should reach a steady state value once the vehicle is in the desired operating mode, i.e., at the desired velocity, $v_{0}$ and desired gap $x_{N, 1}^{*}$.

Figure 4 illustrates how output rise time varies with $n$ and $\delta$ for $N=50$ vehicles. All vehicles are initially at rest in an equilibrium state, with the exception of the leader, which has an initial velocity of $v_{0}$. It is clear that $n$ has an effect on the rise time and that there exists an $n$ where the rise time is minimized, just like in the earlier mass-spring-damper simulation. It also illustrates how the rise time is affected by the parameter, $\delta$, and how the optimal number for $n$ varies with $\delta$, the acceleration constant. In this example, for $\delta<2$ the trend seems to be that the minimum rise time is realized at $n=1$ with the rise time increasing with increasing $n$. However with $\delta \geq 2$ the relationship changes, and the rise time is minimized at some value $n>1$ as seen earlier. The results shown above suggest that this $n$-neighbor coupling may have applications in improving traffic flow.

\section{CONCLUSION}

Increasing the algebraic connectivity of a network increases the rate of convergence of a dynamical system evolving on the network to steady-state (e.g., to consensus). This paper provides new insight into the role of the algebraic connectivity in the transient response of a distributed system. Specifically, we use an idealized one-dimensional model of 


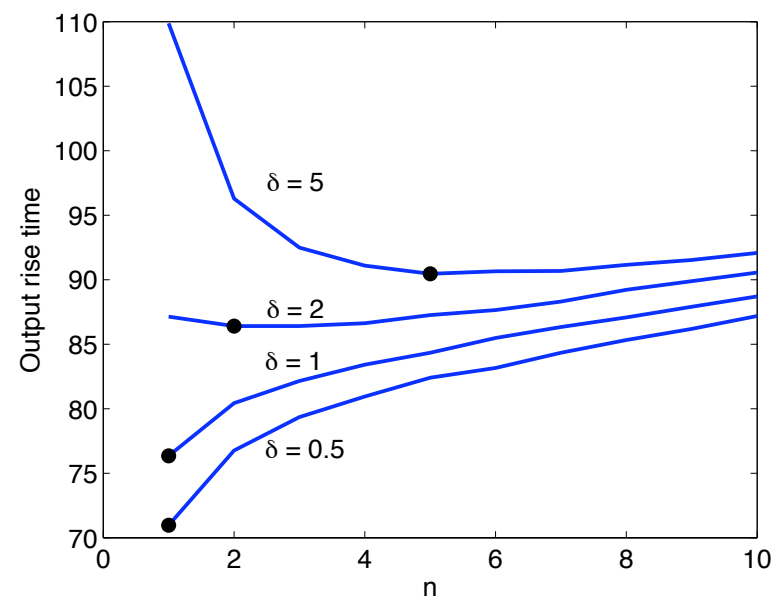

Fig. 4. Simulation results for the modified Intelligent Driver Model with 50 vehicles. The output rise time is minimized at $n=1$ when $\delta<2$. For $2 \leq \delta \leq 5$, the rise time is minimized at some $1<n \ll N$ (black dots).

an interaction network to identify an upper bound on the algebraic connectivity that ensures the transient response is not overdamped. The upper bound is inversely proportional to the squared damping ratio of the node-level interactions. Networks that maximize the algebraic connectivity subject to this constraint achieve a critically damped response. Simulations show that a critically damped response minimizes the output rise time in an $n$-neighbor network, including in an extended version of the Intelligent Driver Model.

In ongoing work we are extending the analysis by constructing a multi-dimensional modeling framework that encompasses nonlinearities in the interactions and the configuration space. We also are working to perform a robustness analysis and to connect this work to string and mesh stability [20].

\section{ACKNOWLEDGMENT}

The author thanks Iain Couzin for sharing his interest in agitation waves in fish schools.

\section{REFERENCES}

[1] D. V. Radakov, Schooling in the ecology of fish. J. Wiley, 1973, translated from Russian by H. Mills.
[2] P. Domenici and R. S. Batty, "Escape behavior of solitary herring (Clupea harengus) and comparisons with schooling individuals," Marine Biology, vol. 128, no. 1, pp. 29-38, 1997.

[3] M. Ballerini, N. Cabibbo, R. Candelier, A. Cavagna, E. Cisbani, I. Giardina, V. Lecornte, A. Orlandi, G. Parisi, A. Procaccini, M. Viale, and Z. Zdravkovic, "Interaction ruling animal collective behavior depends on topological rather than metric distance: Evidence from a field study," Proc. National Academy of Sciences, vol. 105, no. 4, pp. 1232-1237, 2008.

[4] L. Moreau, "Stability of multiagent systems with time-dependent communication links," IEEE Trans. Automatic Control, vol. 50, no. 2, pp. 169-182, 2005.

[5] W. Ren and R. W. Beard, "Consensus seeking in multiagent systems under dynamically changing interaction topologies," IEEE Trans. Automatic Control, vol. 50, no. 5, pp. 655-661, 2005.

[6] R. Olfati-Saber and R. M. Murray, "Consensus problems in networks of agents with switching topology and time-delays," IEEE Trans. Automatic Control, vol. 49, no. 9, pp. 1520-1533, 2004.

[7] R. Olfati-Saber, "Ultrafast consensus in small-world networks," in Proc. 2005 Amer. Control Conf., Portland, Oregon, June 2005, pp. 2371-2378.

[8] S. Boyd, P. Diaconis, and L. Xiao, "The fastest mixing Markov chain on a graph," SIAM Review, vol. 46, no. 4, pp. 667-689, 2004.

[9] J. Sun, S. Boyd, L. Xiao, and P. Diaconis, "The fastest mixing Markov process on a graph and a connection to a maximum variance unfolding problem," SIAM Review, vol. 48, no. 4, pp. 681-699, 2006.

[10] R. Sepulchre, D. A. Paley, and N. E. Leonard, "Stabilization of planar collective motion: All-to-all communication," IEEE Trans. Automatic Control, vol. 52, no. 5, pp. 811-824, 2007.

[11] _ "Stabilization of planar collective motion with limited communication," IEEE Trans. Automatic Control, vol. 53, no. 3, pp. 706-719, 2008.

[12] D. A. Paley, "Stabilization of Collective Motion on a Sphere," Automatica, vol. 45, no. 1, pp. 212-216, 2009.

[13] D. A. Paley and C. Peterson, "Stabilization of Collective Motion in a Time-Invariant Flowfield," J. Guidance, Control, and Dynamics, vol. 32, no. 3, pp. 771-779, 2009.

[14] M. Treiber, A. Hennecke, and D. Helbing, "Congested traffic states in empirical observations and microscopic simulations," Phys. Rev. E, vol. 62 , no. 2 A, pp. $1805-1824,2000$.

[15] W. Ren, "On consensus algorithms for double-integrator dynamics," IEEE Trans. Automatic Control, vol. 53, no. 6, pp. 1503-1509, 2008.

[16] R. Merris, "Laplacian matrices of graphs: A survey," Linear Algebra and its Applications, vol. 197, no. 198, pp. 143-176, 1994.

[17] R. M. Gray, "Toeplitz and circulant matrices: A review," Foundations and Trends in Communications and Information Theory, vol. 2, no. 3, pp. 155-239, 2006.

[18] R. Olfati-Saber, "Algebraic connectivity ratio of Ramanujan graphs," in Proc. 2007 American Control Conf., New York, New York, July 2007, pp. 4619-4624.

[19] E. W. Weisstein. "Cubic Formula." From MathworldA Wolfram Web Resource. [Online]. Available: http://mathworld.wolfram.com/CubicFormula.html

[20] A. Pant, P. Seiler, T. J. Koo, and K. Hedrick, "Mesh stability of unmanned aerial vehicle clusters," in Proc. Amer. Cont. Conf., Arlington, Virginia, June 2001, pp. 62-68. 